\title{
Elementary Proof for Fermat' S Last Theorem 01-01-2010
}

\author{
S. Haridasan, \\ Kadayil House, Pampuram, Kalluvathukkal P.O, Kollam, Kerala,India.Pin:691578 .
}

\section{Introduction}

The famous Fermat's Last Theorem was proved, after three and a half centuries, by Prof: Andrew Wiles and his associate Prof: Richard Taylor in 1994. It is highly advanced. There is search for a simple proof. Congruence modulo addition and multiplication theorems, which are common text book matters, comes to our help. This proof, if found valid, offers very simple one that can be understood by UG students as well.

\section{Fermat's last theorem .}

There is no solution for $a^{n}+b^{n}=c^{n}$ for $n>2$ and $a, b, c$ integers $>0$

\section{Proof}

First we may try to prove single digit solutions using congruence relations for nonzero positive integers.

\subsection{Congruence Modulo addition}

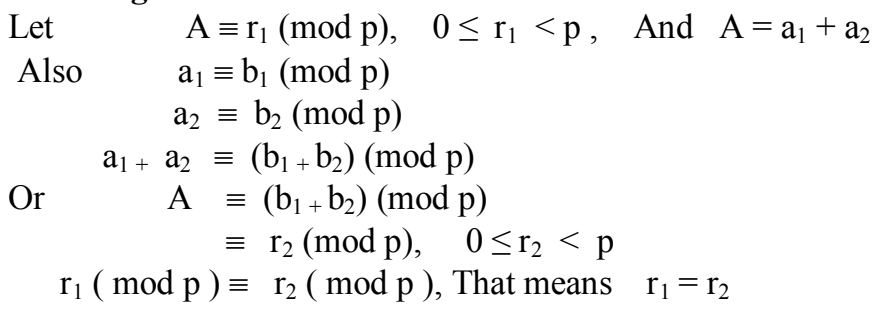

Least Residue before expansion is equal to Least Residue after expansion in Congruence modulo addition for the same $(\bmod p)$.

Ex: (i)

$$
\begin{array}{cc}
35 & =25+10 \\
35 & \equiv 3(\bmod 8) \\
25 & \equiv 1(\bmod 8) \\
10 & \equiv 2(\bmod 8) \\
25+10 \equiv(1+2)(\bmod 8) \\
35 & \equiv 3(\bmod 8) \\
3(\bmod 8) \equiv 3(\bmod 8)
\end{array}
$$

Therefore the Least Residues are equal

\subsection{Congruence modulo Multiplication}

Let $\quad A \equiv r_{1}(\bmod p), 0 \leq r_{1}<p$

And $\quad A=a_{1} \cdot a 2$

Also $\quad a_{1} \equiv b_{1}(\bmod p), \quad 0 \leq b_{1}<p$

$\mathrm{a}_{2} \equiv \mathrm{b}_{2}(\bmod \mathrm{p}), \quad 0 \leq \mathrm{b}_{2}<\mathrm{p}$

$\mathrm{a}_{1} \cdot \mathrm{a}_{2} \equiv\left(\mathrm{b}_{1} \cdot \mathrm{b}_{2}\right)(\bmod \mathrm{p})$

$\mathrm{A} \equiv \mathrm{r}_{2}(\bmod \mathrm{p}), 0 \leq \mathrm{r}_{2}<\mathrm{p}$

$\mathrm{r}_{1}(\bmod \mathrm{p}) \equiv \mathrm{r}_{2}(\bmod \mathrm{p})$, So $\mathrm{r}_{1}=\mathrm{r}_{2}$

For Congruence modulo Multiplication also Least Residue before expansion is equal to Least Residue after expansion for same $(\bmod p)$

$\begin{array}{rll}\text { Ex :(ii) } & 143 & \equiv 13 \times 11 \\ 143 & \equiv 7(\bmod 8) \\ 13 & \equiv 5(\bmod 8) \\ 11 & \equiv 3(\bmod 8) \\ 13 \times 11 & \equiv(5 \times 3)(\bmod 8) \\ 143 & \equiv 15(\bmod 8) \\ & \equiv 7(\bmod 8)\end{array}$


$7(\bmod 8) \equiv 7(\bmod 8)$

Therefore the Least Residues are equal

\subsection{General case for single digits}

$$
\begin{aligned}
3^{3} & \equiv 3(\bmod 8) \\
3^{2} & \equiv 1(\bmod 8) \\
3^{3} \times 3^{2} & \equiv(3 \times 1)(\bmod 8) \\
3^{5} & \equiv 3(\bmod 8)
\end{aligned}
$$

Again $3^{5} \times 3^{2} \equiv(3 \times 1)(\bmod 8)$

$$
3^{7} \quad \equiv 3(\bmod 8)
$$$$
\Rightarrow x^{n} \quad \equiv x(\bmod 8) \text { for all } x \text { odd integers, } n \text { odd integers } \geq 3
$$

Also

$$
\begin{aligned}
4^{3} & \equiv 0(\bmod 8) \\
4^{2} & \equiv 0(\bmod 8)
\end{aligned}
$$

$4^{3} \times 4^{2} \equiv(0 \times 0)(\bmod 8)$

$$
4^{5} \quad \equiv 0(\bmod 8)
$$

$4^{5} \times 4^{2} \equiv(0 \times 0)(\bmod 8)$

$$
4^{7} \quad \equiv 0(\bmod 8)
$$

$\Rightarrow y^{n} \quad \equiv 0(\bmod 8)$, for all $y$ even integers, $n$ odd integers $\geq 3$.

Now for $n=3,5,7,9 \ldots$ odd powers

$$
\begin{array}{ll}
1^{\mathrm{n}} & \equiv 1(\bmod 8) \\
2^{\mathrm{n}} & \equiv 0(\bmod 8) \\
3^{\mathrm{n}} & \equiv 3(\bmod 8) \\
4^{\mathrm{n}} & \equiv 0(\bmod 8) \\
5^{\mathrm{n}} & \equiv 5(\bmod 8) \\
6^{\mathrm{n}} & \equiv 0(\bmod 8) \\
7^{\mathrm{n}} & \equiv 7(\bmod 8) \\
8^{\mathrm{n}} & \equiv 0(\bmod 8) \\
9^{\mathrm{n}} & \equiv 1(\bmod 8)
\end{array}
$$

Hence the TABLE [1] below

\subsection{Proposition I}

For any triplet of the form $\mathrm{a}^{2}+\mathrm{b}^{2}=\mathrm{c}^{2}$ this congruence modulo test satisfies. It can be $(\bmod 9),(\bmod 8)$, $(\bmod 7)$ etc.

Ex: (iii) $\quad 25^{2}=24^{2}+7^{2}$

$$
\begin{gathered}
25^{2} \equiv 1(\bmod 8) \\
24^{2} \equiv 0(\bmod 8) \\
7^{2} \equiv 1(\bmod 8)
\end{gathered}
$$

By 3.1

$$
\begin{aligned}
& 24^{2}+7^{2} \equiv(0+1)(\bmod 8) \\
& 25^{2} \equiv 1(\bmod 8)
\end{aligned}
$$

$1(\bmod 8) \equiv 1(\bmod 8)$

Here the Least Residue parts are equal

If $a^{2}+b^{2}=c^{2}$ is a solution for $a, b, c>0$ and co-prime, we have

$$
\begin{aligned}
\mathrm{a}^{2} & \equiv \mathrm{a}_{1}(\bmod \mathrm{p}) \\
\mathrm{b}^{2} & \equiv \mathrm{b}_{1}(\bmod \mathrm{p}) \\
\mathrm{c}^{2} & \equiv \mathrm{c}_{1}(\bmod \mathrm{p})
\end{aligned}
$$

Then $\left(a_{1}+b_{1}\right)(\bmod p) \equiv c_{1}(\bmod p)$

$\begin{aligned} \mathrm{r}_{1}(\bmod \mathrm{p}) & \equiv \mathrm{c}_{1}(\bmod \mathrm{p}) \\ \text { That means } & \mathrm{r}_{1}=\mathrm{c}_{1} \quad[\mathrm{by} 3.1]\end{aligned}$

$\mathrm{r}_{1}, \mathrm{c}_{1}$ being the Least Residues

In general if $a^{n}+b^{n}=c^{n}$ has a solution for $n>2$ then it should satisfy the congruence modulo test.

\subsection{Proposition II}

$$
\text { If } \begin{aligned}
x & =y+z \quad \text { where } \quad x, y, z \neq 0 \\
x^{2} & =(y+z)^{2}=y^{2}+2 y z+z^{2} \text {, then } x^{2} \neq y^{2}+z^{2}
\end{aligned}
$$

Similarly when $x=y+z$, then $x^{n} \neq y^{n}+z^{n}$ 


\subsection{Proposition III}

For non zero integers $\mathrm{x}, \mathrm{y}$

$$
1^{\mathrm{n}}+\mathrm{x}^{\mathrm{n}} \neq \mathrm{y}^{\mathrm{n}}
$$

With the help of these propositions we can verify, from the table [1] that for any a, b, c non zero integers and co-prime, $\mathrm{a}^{\mathrm{n}}+\mathrm{b}^{\mathrm{n}}=\mathrm{c}^{\mathrm{n}}$ has no single digit solutions for $\mathrm{n}=3,5,7,9, \ldots$ odd powers.

Verification from TABLE [1], $1^{\text {st }}$ and $3^{\text {rd }}$ rows:

\begin{tabular}{llll}
$\begin{array}{l}\text { Numbers } \\
1^{\mathrm{n}}+2^{\mathrm{n}} \text { etc }\end{array}$ & Residues & $\begin{array}{c}\text { Solutions } \\
\text { NIL }\end{array}$ & $\begin{array}{c}\text { Reason } \\
\text { Prop. III }\end{array}$ \\
\hline $2^{\mathrm{n}}+3^{\mathrm{n}}$ & $0+3 \Rightarrow 3$ & NIL & \\
$2^{\mathrm{n}}+4^{\mathrm{n}}$ & $0+0 \Rightarrow 0$ & NIL & Co-prime \\
$2^{\mathrm{n}}+5^{\mathrm{n}}$ & $0+5 \Rightarrow 5$ & NIL & Co-prime \\
$2^{\mathrm{n}}+6^{\mathrm{n}}$ & $0+0 \Rightarrow 0$ & NIL & NIL \\
$2^{\mathrm{n}}+7^{\mathrm{n}}$ & $0+7 \Rightarrow 7$ & NIL & Co-prime \\
$2^{\mathrm{n}}+8^{\mathrm{n}}$ & $0+0 \Rightarrow 0$ & NIL & \\
$2^{\mathrm{n}}+9^{\mathrm{n}}$ & $0+1 \Rightarrow 1$ & NIL & \\
\hline $3^{\mathrm{n}}+4^{\mathrm{n}}$ & $3+0 \Rightarrow 3$ & Prop II \\
$3^{\mathrm{n}}+5^{\mathrm{n}}$ & $3+5 \Rightarrow 8 \Rightarrow 0$ & NIL & \\
$3^{\mathrm{n}}+6^{\mathrm{n}}$ & $3+0 \Rightarrow 3$ & NIL & \\
$3^{\mathrm{n}}+7^{\mathrm{n}}$ & $3+7 \Rightarrow 10 \Rightarrow 2$ & NIL & \\
$3^{\mathrm{n}}+8^{\mathrm{n}}$ & $3+0 \Rightarrow 3$ & NIL & \\
$3^{\mathrm{n}}+9^{\mathrm{n}}$ & $3+1 \Rightarrow 4$ & NIL & Co-prime \\
\hline $4^{\mathrm{n}}+5^{\mathrm{n}}$ & $0+5 \Rightarrow 5$ & NIL & \\
$4^{\mathrm{n}}+6^{\mathrm{n}}$ & $0+0 \Rightarrow 0$ & NIL & \\
$4^{\mathrm{n}}+7^{\mathrm{n}}$ & $0+7 \Rightarrow 7$ & NIL & \\
$4^{\mathrm{n}}+8^{\mathrm{n}}$ & $0+0 \Rightarrow 0$ & NIL & \\
$4^{\mathrm{n}}+9^{\mathrm{n}}$ & $0+1 \Rightarrow 1$ & NIL & \\
\hline $5^{\mathrm{n}}+6^{\mathrm{n}}$ & $5+0 \Rightarrow 5$ & NIL & \\
$5^{\mathrm{n}}+7^{\mathrm{n}}$ & $5+7 \Rightarrow 12 \Rightarrow 4$ & NIL & \\
$5^{\mathrm{n}}+8^{\mathrm{n}}$ & $5+0 \Rightarrow 5$ & NIL & \\
$5^{\mathrm{n}}+9^{\mathrm{n}}$ & $5+1 \Rightarrow 6$ & NIL & \\
\hline $6^{\mathrm{n}}+7^{\mathrm{n}}$ & $0+7 \Rightarrow 7$ & NIL & \\
$6^{\mathrm{n}}+8^{\mathrm{n}}$ & $0+0 \Rightarrow 0$ & NIL & NIL \\
$6^{\mathrm{n}}+9^{\mathrm{n}}$ & $0+1 \Rightarrow 1$ & NIL & \\
\hline $7^{\mathrm{n}}+8^{\mathrm{n}}$ & $7+0 \Rightarrow 7$ & NIL & \\
$7^{\mathrm{n}}+9^{\mathrm{n}}$ & $7+1 \Rightarrow 8 \Rightarrow 0$ & NIL & \\
$8^{\mathrm{n}}+9^{\mathrm{n}}$ & $0+1 \Rightarrow 1$ & & \\
\hline & & &
\end{tabular}

Hence there is no single digit solution for $a^{n}+b^{n}=c^{n}, n=3,5,7,9, \ldots$ odd powers. It is enough to prove for prime exponents greater than 2.

\section{Acknowledgement}

I am extremely grateful to Dr.Thrivikraman, Former Professor and Head of Department of Mathematics, Cochin University (CUSAT) for the valuable suggestions and guidance and Dr.M.I.Jinnah , visiting Professor IISER Thiruvananthapuram for the precise comments that helped me to present this article.

Table [1]

\begin{tabular}{|l|l|l|l|l|l|l|l|l|l|l|l|l|l|l|l|l|}
\hline NUMBERS & 1 & 2 & 3 & 4 & 5 & 6 & 7 & 8 & 9 & & P & 0 & W & E & R & S \\
\hline & 1 & 4 & 1 & 0 & 1 & 4 & 1 & 0 & 1 & & 2 & & & & & \\
\hline RESIDUES & 1 & 0 & 3 & 0 & 5 & 0 & 7 & 0 & 1 & & 3 & 5 & 7 & 9 & 11 & $\ldots$ \\
\hline & 1 & 0 & 1 & 0 & 1 & 0 & 1 & 0 & 1 & & 4 & 6 & 8 & 10 & 12 & $\ldots$ \\
\hline
\end{tabular}

